\title{
DNS of a variable density jet in the supercritical thermodynamic state
}

\author{
Battista F. and Picano F. and Troiani G. and Casciola C.M.
}

\section{Introduction}

Cryogenic rocket engines, advanced gas turbines and diesel engines are characterized by the injection of a liquid fuel into a high temperature and pressure chamber. Typically the fuel is injected at high enough pressure to be close or above the critical pressure. In these conditions the behavior of the fluid differs strongly from that of a perfect gas. It exhibits large variations of thermodynamic and transport properties also for small temperature changes, with significant effects on mixing and combustion processes. In this context an appropriate numerical simulation should take into account such thermodynamic phenomena via suitable equation of state and transport properties relations.

In literature this issue is typically addressed in several experimental studies of round jets at super-critical pressure see [1,2,3]. In sub-critical conditions the mixing/evaporation process leads to the formation of ligaments which break-up in evaporating droplets, while in super-critical conditions the ligaments dissolve in the external environment. In the context of numerical simulations few Large Eddy Simulations (LES) of jet under super-critical conditions have been performed. In particular Zong et al. [4] perform a $2 D$ large eddy simulation reproducing the thermodynamic conditions of the experimental study provided by Mayer et al. [1]. The same conditions are matched in the $3 D$ large eddy simulation carried out by Schmitt et al. [5].

Battista F. Dipartimento di meccanica ed aeronautica "Sapienza" University of Rome, e-mail: francesco.battista@uniroma1.it

Picano F. Dipartimento di meccanica ed aeronautica "Sapienza" University of Rome, e-mail: francesco.picano@uniroma1.it

Troiani G. ENEA C.R. Casaccia, via Anguillarese 301, 00123 Roma Italy, e-mail: guido.troiani@enea.it

Casciola C.M. Dipartimento di meccanica ed aeronautica "Sapienza" University of Rome, e-mail:

carlomassimo.casciola@uniroma1.it 
Moreover an extensive review containing the main results obtained before 2006 is provided in [6].

Aim of this work is to perform a $3 D$ Direct Numerical Simulation of a cryogenic jet at weakly supercritical pressure injected in an environment with the same pressure and ambient temperature. Actually the injection and mixing of cryogenic gases occur with characteristic velocities smaller than the local sound speed. To this purpose, an asymptotic Low Mach number formulation of the Navier-Stokes equations coupled with the Van der Waals state equation is derived to perform the simulations.

\section{Methodology}

The simulations involve a jet injected with a density smaller than the critical one, $\rho_{j}<\rho_{c}$, in an environment at weakly super-critical pressure with density $\rho_{e x t}>\rho_{c}$.

In these conditions the perfect gas assumption is not consistent with the thermodynamics of dense gases near the critical point. Here the Van der Waals equation of state (EOS) is used to mimic the supercritical gas,

$$
\left(p+a^{\prime} \rho^{2}\right)\left(1-b^{\prime} \rho\right)=\rho \theta
$$

where the EOS is expressed in its dimensionless form and $p, \rho$ and $\theta$ are the pressure, density and temperature of the gas, respectively. The constants $a^{\prime}$ and $b^{\prime}$ depend on the attraction forces between molecules and on their average volume occupied, respectively. Since the Van der Waals constants are function of the critical thermodynamic variables, they are expressed in terms of the ratio between the critical and reference (indicated with the subscript $\infty$ ) quantities, $a^{\prime}=3\left(\rho_{\infty} / \rho_{c}\right)\left(p_{c} / p_{\infty}\right)$ and $b^{\prime}=(1 / 3)\left(\rho_{\infty} / \rho_{c}\right)$. The thermodynamic properties, such as enthalpy $\rho h$, internal energy $\rho e$ and ratio of the constant-pressure to constant-volume specific heats $\gamma$, follow by the standard statistical mechanics arguments,

Fig. 1 Snapshot of normalized temperature $\left(\theta-\theta_{\text {ext }}\right) /\left(\theta_{j}-\theta_{\text {ext }}\right)$ field of the three simulations. From left: real gas with $\rho_{j} / \rho_{\text {ext }} \simeq 10$., perfect gas with $\rho_{j} / \rho_{\text {ext }} \simeq 4$., perfect gas with $\rho_{j} / \rho_{\text {ext }} \simeq 10$..

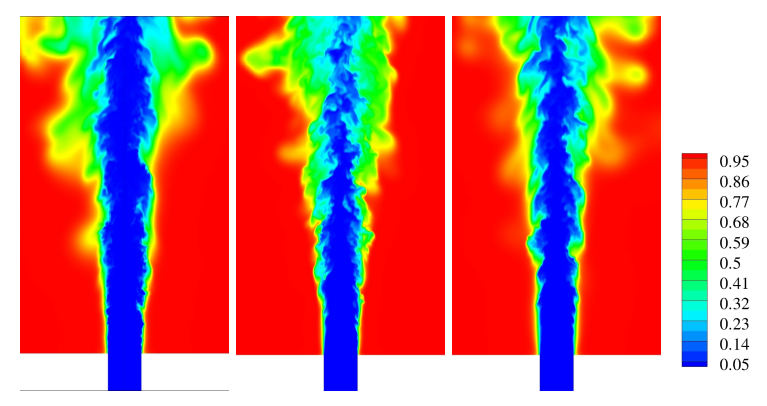




$$
\begin{array}{r}
\rho e=\frac{1}{\gamma-1} \rho \theta-a^{\prime} \rho^{2} \quad \rho h=\rho e+p \\
\gamma=\gamma^{p g}\left[\frac{3}{5}+\frac{2}{5} \frac{1}{1-2 a^{\prime} \frac{\rho}{\theta}\left(1-b^{\prime} \rho\right)^{2}}\right]
\end{array}
$$

where $\gamma^{p g}$ denotes the specific heat ratio of the perfect gas.

The present derivation of the Low Mach number formulation of the NavierStokes equations for a Van der Waals EOS is an extension of that proposed by Majda \& Sethian [7], for the ideal gas. This approach is particularly attractive in the simulation of cryogenic flows that, despite at small Mach number, cannot be considered strictly incompressible due to the occurrence of strong density gradients $[7,8]$. The zeroth-order dimensionless system reads,

$$
\begin{gathered}
\frac{\partial \rho_{0}}{\partial t}+\nabla \cdot(\rho u)_{0}=0 \\
\frac{\partial(\rho u)_{0}}{\partial t}+\nabla \cdot\left[(\rho u)_{0} u_{0}\right]=\frac{1}{R e} \nabla \cdot \tau_{0}-\nabla p_{2}+\rho_{0} f_{0} \\
\nabla \cdot u_{0}=\frac{1}{p_{0}}\left[\frac{1}{\operatorname{RePr} r} \nabla \cdot(\mu \nabla \theta)_{0}\right]\left[\frac{1}{1+\frac{(\gamma-2) a^{\prime} \rho_{0}^{2}}{p_{0} \gamma}+\frac{2 a^{\prime} b^{\prime} \rho_{0}^{3}}{p_{0} \gamma}}\right] \\
\theta_{0}=\frac{p_{0}}{\rho_{0}}\left(1+a^{\prime} \frac{\rho_{0}^{2}}{p_{0}}\right)\left(1-b^{\prime} \rho_{0}\right)
\end{gathered}
$$

where continuity (2) and momentum (3) equations coincide with those of the ideal gas. Energy (4) and EOS (5) equations present instead corrective terms with respect to the real gas behavior. Clearly, the perfect gas formulation is recovered in the limit $a^{\prime} \rightarrow 0$ and $b^{\prime} \rightarrow 0$. The second-order pressure term $p_{2}$ provides the dynamic pressure which is the only pressure term present in the incompressible formulation. In the present context this degree of freedom allows a prescribed value of the velocity divergence, eq. (4), as it happens in the original ideal gas formulation [7].

As anticipated our purpose here is to perform a $3 D$ Direct Numerical Simulation (DNS) of a turbulent jet based on the above equations. The Eulerian algorithm discretizes the system in a cylindrical domain to address a variable density jet at weakly supercritical conditions. Spatial discretization is based on central second order finite differences in conservative form on a staggered grid while the convective term of scalar equations is dealt with by a bounded central difference scheme to avoid spurious oscillations. Temporal evolution is performed by a low-storage third order Runge-Kutta scheme. Dirichlet (prescribed velocity) conditions are enforced at the inflow, by using a cross-sectional plane of a periodic turbulent pipe flow, obtained by a companion time-evolving DNS. A convective condition is adopted at the outflow, while a traction-free condition is used for the side boundary. More details on the code are available in [9]. 
Three simulations are performed. The first one reproduces a cold jet of real gas $(S 1)$ injected in a environment with a pressure weakly supercritical $p / p_{c} \simeq 1.18$. The density of the environment is one tenth the jet one $\rho_{j} / \rho_{\text {ext }}=10$, ensuring that $\rho_{j}<$ $\rho_{c}<\rho_{\text {ext }}$, while the ratio between the jet and environment temperature is $\theta_{\text {ext }} / \theta_{j} \simeq$ 4.. The other two simulations $S 2, S 3$ reproduce two perfect gas jets matching either the temperature ratio $\theta_{\text {ext }} / \theta_{j}=\rho_{j} / \rho_{\text {ext }}=4$ or the density ratio $\theta_{\text {ext }} / \theta_{j}=\rho_{j} / \rho_{\text {ext }}=$ 10 of the first one, respectively. All simulations present a Reynolds number based on the jet diameter, equal to $R e_{D}=U_{0} D / \nu_{\infty}=6000$, with $U_{0}$ the bulk velocity. The thermodynamic parameters mimic one of the experiments with the cold jet of nitrogen in nitrogen environment performed by Mayer et al. in [1].

The computational domain, $\left[\phi_{\max } \times R_{\max } \times Z_{\max }\right]=[2 \pi \times 6.2 D \times 10 D]$ is discretized by $N_{\phi} \times N_{r} \times N_{z}=128 \times 201 \times 600$ nodes with a stretched mesh in the radial direction to assure a resolved shear layer. The grid size in the jet region is about two/three times the Kolmogorov scale, and is able to accurately capture the strong density gradients which develops in the flow, [10]. After reaching the statistical steady state, about two hundred complete fields, separated by $0.125 \mathrm{D} / U_{0}$, are collected for statistical analysis.

In the figure 1 the instantaneous field of normalized temperature is presented for the three simulations. Apparently the density ratio has a larger influence on the jet than the temperature ratio. The core of the jet in the $S 2$ case (middle panel) presents in fact a temperature lower that the real gas jet (left panel). Also the perfect gas jet (right panel) with the same density ratio (S3) of the real gas flow, has an instantaneous temperature lower though the difference is sensibly smaller than the previous case $S 2$ vs $S 1$. The temperature difference in the latter case are probably associated with the thermodynamic properties of the real gas near the critical point.

\section{Results}

Figure 2 reports the axial profiles of the Reynolds averaged centerline density (left panel), temperature (middle panel) and velocity (right panel). These mean quantities
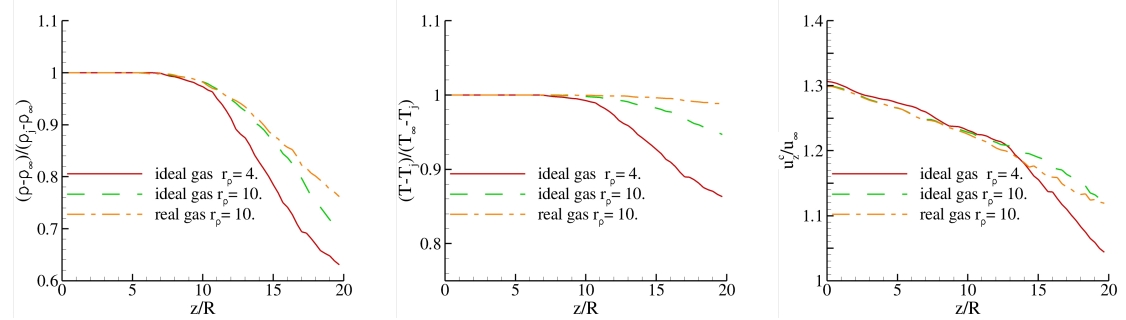

Fig. 2 Mean axial profiles of density (left panel) normalized with the injection and surroundings density $\left(\rho-\rho_{j}\right) /\left(\rho_{e x t}-\rho_{j}\right)$, normalized centerline temperature (middle panel) $\left(\theta-\theta_{\text {ext }}\right) /\left(\theta_{j}-\right.$ $\left.\theta_{\text {ext }}\right)$ and normalized centerline axial velocity $u_{z}^{c} / u_{\infty}$ 

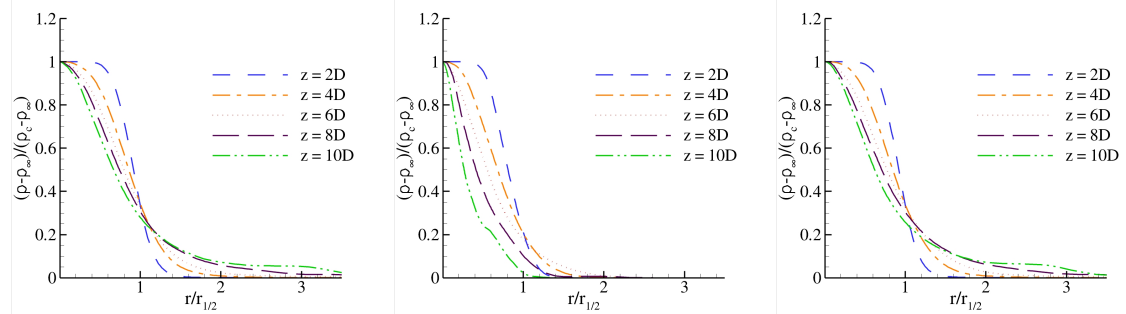

Fig. 3 Mean radial profiles of density normalized with the centerline and surroundings density, $\left(\rho-\rho_{\text {ext }}\right) /\left(\rho_{c}-\rho_{\text {ext }}\right)$. The radial coordinate is normalized with the radial distance where the averaged velocity is the half of the corresponding centerline value. From left: real gas with $\rho_{j} / \rho_{\text {ext }} \simeq 10$., perfect gas with $\rho_{j} / \rho_{\text {ext }} \simeq 4$., perfect gas with $\rho_{j} / \rho_{\text {ext }} \simeq 10$..

confirm that the density ratio determines the jet dynamics more than the temperature ratio. The decay of density and of the normalized temperature is more rapid in the configuration $S 2$. The behavior in the dense-gas simulation, $S 1$, and for the ideal gas at matching density ratio, $S 3$, is quite similar although in the far field the ideal gas jet presents a weaker reduction of both density and temperature. In the velocity plots, for $S 2$ a curve slope variation occurs at $z \simeq 12 D$, while the other jets show a liquid-like behavior. Figures 3 and 4 provide the mean density and velocity profiles, respectively, as a function of the radial coordinate at five different axial locations. Mean quantities are normalized by their centerline and surroundings values, while the radial coordinate is normalized by the jet half-width $r_{1 / 2}$, radial distance where the mean axial velocity is the half of the corresponding centerline value. The density profiles, figure 3 , highlights one more that the the density ratio plays a crucial role in the jet dynamics. Actually the simulations $S 1$ and $S 3$ exhibit a similar behavior also of the radial profiles, while the $S 2$ differs considerably. In addition, only the density profiles of $S 1$ and $S 3$ seem to collapse on a unique self-similar curve at small axial distances, $z=8-10 D$, presenting noticeable differences for radial distances larger
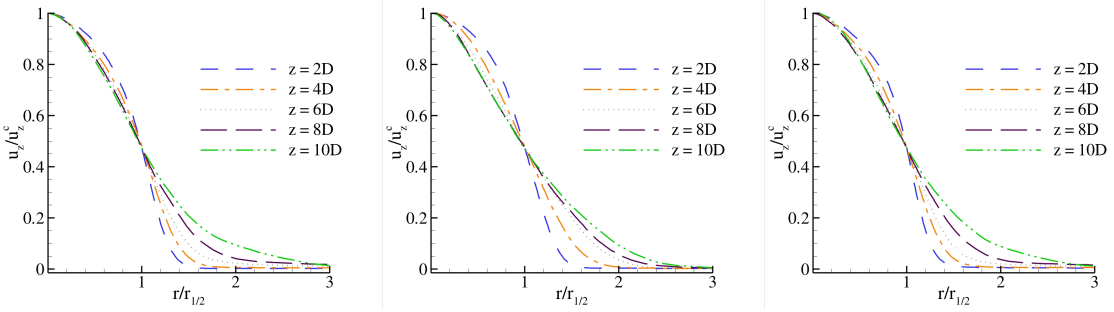

Fig. 4 Mean radial profiles of axial velocity normalized with the centerline mean velocity, $u_{z} / u_{z}^{c}$. The radial coordinate is normalized with the radial distance where the averaged velocity is the half of the corresponding centerline value. From left: real gas with $\rho_{j} / \rho_{\text {ext }} \simeq 10$., perfect gas with $\rho_{j} / \rho_{\text {ext }} \simeq 4$., perfect gas with $\rho_{j} / \rho_{\text {ext }} \simeq 10$. 
than a couple of $r_{1 / 2}$. The existence of self-similarity is observed also in the $2 D$ large eddy simulation provided in [4] even if at axial distances quite larger than the present distances. The axial velocity profiles, figure 4 , tend to collapse on a unique self-similar curve in all jets, also for that with the density ratio of 4 ( $S 2$, middle panel). Nonetheless also for the velocity appreciable differences emerge at the edge of the jet, more than $r_{1 / 2}$.

\section{Conclusions}

A numerical algorithm to deal with Low-Mach number supercritical flows is proposed and used to perform a DNS of real gas turbulent jet in cryogenic conditions. The effect of the real gas EOS is analyzed comparing the results with two simulations of ideal gas matching the density ratio in the first and the temperature ratio in the other. The real gas jet presents a liquid-like behavior showing a density/temperature core less influenced by the surrounding environment, in respect to ideal jets. Hence the mixing in real gas jets appear reduced in respect to ideal conditions. Besides the mean axial velocity and density profiles show a tendency to a self-similar behavior as in the incompressible limit. Nonetheless difference in the outer region of the jet emerges that could disappear at larger axial distances.

Acknowledgements The work was supported by the Standard HPC-2010 Grant n. std10-284 for providing CPU time and storage at CASPUR High Performance Computing center.

\section{References}

1. Mayer, W., Schik, A., Schweitzer, C. \& Schaffler, M. (1995). Injection and mixing processes in high pressure LOX/GH2 rocket combustion. AIAA 16

2. Chehroudi, B., Tally, D. \& Coy, E. (2002). Visual characteristics and initial growth rate of round cryogenic jets at sub-critical and super-critical pressures. Physic of Fluids 14: 850-861

3. Oschwald, M. (1999).Supercritical nitrogen free jet investigated by spontaneous Raman scattering. Experiments in Fluids 27:497-506

4. Zong, N., Meng, H., Hsieh, S.Y. \& Yang, V. (2004). A numerical study of cryogenic fluid injection and mixing under supercritical conditions. Physics of fluids 16:4248-4261

5. Schmitt, T., Selle, L., Cuenot, B. \& Poinsot, T. (2009).Large-Eddy Simulation of transcritical flows. Comptes Rendus Mécanique 337, 6-7:528-538

6. Zong, N. \& Yang, V. (2006). Cryogenic fluid jets and mixing layers in trans-critical and supercritical environments. Comb. Science and Technology 178:193-227

7. Majda, A. \& Sethian, J. (1985). The derivation and numerical solution of the equations for zero Mach number combustion. Combustion science and technology 42, 3:185-205

8. Müller, B.(1998). Low-Mach-number asymptotics of the Navier-Stokes equations. Journal of Engineering Mathematics 34,1:97-109

9. Picano, F. \& Casciola, C.M. (2007). Small-scale isotropy and universality of axisymmetric jets. Physics of fluids 19:118106

10. Picano, F., Battista, F., Troiani, G. \& Casciola, C.M. (2010). Dynamics of PIV seeding particles in turbulent premixed flames. Experiments in Fluids, doi:10.1007/s00348-010-0896-y 